\title{
COMMUNICATION AND INTERACTION FROM FACE-TO-FACE TO ONLINE EMI DEGREE PROGRAMMES IN THE STUDENTS' PERSPECTIVE - A CASE STUDY
}

\author{
Stefania Cicillini, Antonella Giacosa, University of Torino, Italy
}

\section{Abstract}

The proliferation of EMI degree programmes, which are completely taught through the medium of English, has risen steadily in Italy over the last two decades, especially at master level. Along with English proficiency, interaction and communication are key factors in the success of EMI programmes even though they have been matter of concern during the COVID-19 emergency, when all classes were suddenly forced to shift online. More specifically, students of EMI degree programmes in "Medicine and Surgery" and "Nursing" were impacted because they used to work and study in groups, to interact with patients and classmates and to receive immediate feedback from lecturers.

Though unplanned, emergency remote education (ERE) has allowed students to attend classes and take exams. However, it has also shown that interaction and communication could have been handled better. First, this study aims to investigate how these issues were dealt with during the emergency; second, it enquires what lessons can be learned from the sudden transition onto digital platforms. After experiencing ERE, 102 students from various Italian universities have filled in an online questionnaire reflecting on how interaction and communication were mediated during online classes or video lessons. On the one hand, their reflections have provided insight into challenges for EMI pedagogy, which had already been identified in the face-to-face modality but were exacerbated during ERE; on the other hand, their suggestions could be useful to improve future EMI classes. Data has shown that specific training in digital tools, standardization of the lessons' format, more interaction and investments in better platforms are considered to be key aspects to implement and promote in EMI courses in the forthcoming years.

Keywords: higher education; English-medium instruction; EMI; online learning; ERE. 
Cicillini, S., \& Giacosa, A.

Communication and Interaction from Face-to-Face to Online EMI Degree Programmes in the Students' Perspective - A Case Study

\section{Introduction}

As a consequence of the globalized and connected world, the proliferation of Englishmedium instruction (EMI) degree programmes, which are completely taught in English, has grown considerably throughout Italy over the last two decades, especially at master level (Wächter \& Mainworm, 2014; Broggini \& Costa, 2017, Cicillini (bis), forthcoming). Due to the increasing interest of universities in attracting international students and the attention of Italian students to have experiences abroad, more EMI degree courses are bound to be implemented in the forthcoming years. However, despite the steadily growing interest, concerns have arisen on the generally low levels of English proficiency, hindered interaction and understanding, and possible impoverishment of quality standards in comparison to courses taught in the students' mother tongue (Costa \& Coleman, 2013; Pulcini \& Campagna, 2015).

Along with English proficiency, interaction and communication are major challenges for the success of EMI degree programmes (Drljača Margic \& Vodopija-Krstanovic, 2018) and have been issues of even more concern during the COVID-19 emergency. In the late winter of 2020, the whole education system was forced to move onto digital platforms and classes, which until then had been taught in person, had to be offered online. Consequently, all students and lecturers had the chance to experience distance learning and all the EMI degree programmes in Italy had to be suddenly transformed into online courses. Although it was emergency remote learning (ERE), a term referring to the unplanned response to an educational emergency (Bozkurt et al., 2020), this massive experience of distance learning has provided interesting insights into EMI pedagogy.

This paper focuses on students of "Medicine and Surgery" and "Nursing" EMI degree programmes and aims to contribute to the discussion about critical aspects of the related pedagogy. More specifically, this study deals with the changes in interaction and communication, which were experienced by students from several Italian universities during the lockdown. In the first part of the present paper, the theoretical framework along with the research questions and methodology will be presented. In the second part, quantitative and qualitative data will be discussed to identify key aspects relating to interaction and communication, which impacted significantly on "Medicine and Surgery" and "Nursing" EMI students during the emergency and could contribute to implementing EMI pedagogy both in online and in-person classes. 
Cicillini, S., \& Giacosa, A.

Communication and Interaction from Face-to-Face to Online EMI Degree Programmes in the Students' Perspective - A Case Study

\section{Theoretical framework}

In this study on EMI degree programmes, communication is intended as the act of giving information and sharing knowledge on a subject matter in a multicultural and internationalized environment and through a common language that is English. Considering the presence of both local and international students with different cultures, mother tongues and backgrounds, intercultural communication (ICC) in EMI classes plays a key role in creating a positive learning environment and conveying the meaning. In such a context, behaviour, body language and language use may influence the learning experience and the quality of communication and interaction (TAEC EMI handbook, 2019).

Interaction, which is intended as the mutual or reciprocal influence and exchange of information is intertwined with communication and as Moore (1989) suggested, it may assume different shapes depending on the people involved. He identified three types of interaction: learner-learner (relies on the collaboration between learners with similar knowledge of a certain discipline); learner-instructor (relies on the exchange between teachers and learners); learner-content (in which students learn from the subject, the materials and references provided) (Moore, 1989; Cicillini \& Salusso, 2019).

In the EMI environment, effective communication and interaction have proved to be challenging and difficult to promote because different cultures and levels of language proficiency coexist within the same class. Given the variety of languages, cultures and learning styles, interaction and communication may be interpreted differently, especially by those students who are not used to interact and to actively participate in class. Dealing with these issues may be particularly challenging also for lecturers who have to deliver specialized content, give feedback and facilitate interaction. Although there is very little published research on interaction at the tertiary level of education in EMI classes (Macaro et al., 2017), some research reports that some lecturers do not feel confident when they have to teach through English and struggle when it comes to answer students' questions and mediate interactions in live classes (Dafouz Milne \& Sanchez Garcia, 2013; Borsetto, \& Schug, 2016). Indeed, the use of questions in EMI classes as a strategy to foster interaction seems to be often avoided by those lecturers who do not feel at ease in communicating through English, mainly for fear to lose face and credibility with their students (Drljaca Margic \& Vodopija-Krstanovic, 2018). Another strategy used by some EMI lecturers to foster interaction is the use of both English and their mother tongue, especially when communication becomes difficult to manage (Macaro et al., 2017). Codeswitching may be an effective solution to overcome linguistic and communication problems in those contexts where all learners speak both the languages used; nevertheless, 
Cicillini, S., \& Giacosa, A.

Communication and Interaction from Face-to-Face to Online EMI Degree Programmes in the Students' Perspective - A Case Study

this may not always be the case of EMI classes which are usually characterized by the presence of local and international students and a certain linguistic diversity.

Communication and interaction play a major role in the digital environment for a successful learning process (Cicillini \& Salusso, 2019). Although the literature on e-learning is wide, for the purposes of the present paper attention will be devoted on the barriers of communication and the different types of interaction identified in distance learning by Moore (1989).

\section{Methodology and Research Questions}

This research focuses on the students' perceptions of the opportunities and challenges emerged during the unplanned shift from in-person to distance learning, identified as ERE (Bozkurt et al., 2020). Specifically, communication and interaction in the EMI degree programmes in "Medicine and Surgery" and "Nursing" are observed starting from the students' perspective.

The research questions this study seeks to answer are the following:

70. How do communication and interaction change in the shift from the face-to-face to the online modality in EMI degree programmes?

71. What lessons can we learn from the sudden shift of EMI courses onto digital platforms due to the coronavirus emergency?

In order to tackle these issues, data collection was conducted through two online questionnaires sent during the COVID-19 emergency; thus, the online modality we refer to is specifically the ERE. The questionnaires, which were drafted using Google forms and addressed to lecturers and students, were anonymous, written in English and sent to the lecturers' emails and shared in several Facebook university groups. The participants and universities identified for this research were students and lecturers of 14 EMI degree programmes. Data was collected from Universitaly.it, an institutional webpage where all the degree programmes in Italian and English are stored and made available by the Ministry of University and Research.

For the purposes of the study, the students' questionnaire only was taken into account and discussed. 102 students filled in the online questionnaire which was composed of 28 questions, both open-ended and close-ended using a five-point Likert scale. Both quantitative and qualitative data were observed and analysed. The qualitative analysis was carried out by using certain keywords such as interaction, communication and English; the students' profiles and comments were given a number from 1 to 102 in order to diversify the participants. 
Cicillini, S., \& Giacosa, A.

Communication and Interaction from Face-to-Face to Online EMI Degree Programmes in the Students' Perspective - A Case Study

\section{Findings and Discussion}

\section{Context of the study}

Both the degree programmes involved in this study have restricted admission procedures and a limited number of places for prospective students, which are regulated by national entry tests and rankings. They also have specific English language entry requirements which range from B1 to B2, according to the CEFR (Common European Framework of Reference for Languages) descriptors (Cicillini, forthcoming; Cicillini (bis), forthcoming). In addition, while "Medicine and Surgery" is a 6-year master degree programme, "Nursing" is a 3-year bachelor. In the academic year 2019/2020 in which this study takes place, while the first semester was offered in the face-to-face modality the second semester was almost entirely held online, through the ERE modality because of the coronavirus outbreak.

The EMI courses offered during the first semester were held in-person in campus and hospitals as in the previous academic years. Interaction took place mostly in person and in class with immediate feedback from both lecturers and students, through work groups and projects, while communication outside the classroom was facilitated by office hours, emails and institutional web pages. By contrast, in the second semester classes, which were initially offered in the face-to-face modality, were suddenly transformed into online courses. Under those circumstances, there was little time to plan, arrange and standardize lessons in the online modality. For this reason, different digital platforms were used to do live classes (synchronous mode) such as Zoom (41\%), Microsoft Teams (20\%) and WebEx (18\%), which were sometimes uploaded to Moodle pages (22\%) after the classes. Besides, other lecturers decided to offer asynchronous classes through pre-recorded videos and PowerPoint presentations. In both these modalities, interaction and communication became more difficult because of technical and organizational issues, especially in the asynchronous classes. As a consequence, most of the interaction and communication took place via digital channels: chats, emails and forums. Learners could communicate with their instructors writing questions in the chat boxes, sending emails or attending online office hours. Moreover, they had to interact with their classmates mostly using their mobile phones or social media as the lockdown rules hindered their usual group activities and study groups. Finally, some of them did not have their study books with them and could rely mostly on digital resources to access study contents. These circumstances have impacted on their learning strategies and challenged them from different points of view as is shown in the following paragraphs. 
Cicillini, S., \& Giacosa, A.

Communication and Interaction from Face-to-Face to Online EMI Degree Programmes in the Students' Perspective - A Case Study

\section{Communication}

As data referring to communication shows, the main issues of concern in students' perceptions are relating to the areas of human contact and the way information was managed.

\section{Human contact}

Considering their answers on what they missed most about face-to-face classes, students stated they were affected by not seeing their classmates and lecturers. Concerning peer communication, they revealed they felt less motivated and had difficulties getting ready for the exams because they could not rely on their work group or teamwork activities (47\%). Regarding communication, students lamented that it was difficult to reach out to them: even if they sent emails to their lecturers (60\%), wrote questions in forums (20\%) to ask for clarifications, used the chat box during online lessons (50\%) and were able to ask for explanations (48\%), they missed the immediate feedback they used to receive during faceto-face classes. Moreover, feeling more anxious about the exams they would have appreciated being reassured and emotionally supported by their lecturers. As data shows, during ERE, socio-emotional aspects were challenging and not always successfully handled. Not all students felt at ease during online classes: for example, $11 \%$ did not dare to ask questions because their class was being recorded. To sum up, this lack of communication increased their difficulties and affected their learning process: not being able to meet their lecturers in-person was not only a socioemotional issue. Students stated they missed the immediate feedback of face-to-face lessons, which allowed mutual understanding and made the explanations clearer. So, it seems necessary to devote more attention to the aspects connected to care, well-being and socioemotional issues by providing specific training to lecturers.

\section{Information and courses organization}

Apart from the human factor, students lamented that they missed clear information about course timetables and exams, as the comment below shows:

"Exam formats and instructions were not clear until just a few days before so it was difficult to prepare properly" (Student 4)

The sudden shift to remote education caused some uncertainties concerning the way classes were taught: some lecturers chose to teach online live classes while others opted for pre-recorded presentations.

"It is important all professors will use the same tools to record a lecture" (Student 88) 
Cicillini, S., \& Giacosa, A.

Communication and Interaction from Face-to-Face to Online EMI Degree Programmes in the Students' Perspective - A Case Study

In their comments, students underlined they could not always rely on clear guidelines and were confused by the fact that lecturers chose different ways to deliver their lessons and did not explain from the beginning of the lockdown how the assessment would be organised.

"I'd prefer if professors formulated a schedule in the beginning and stuck to it rather than upload the lectures whenever they liked or all in one go. Some flexibility is allowed but not knowing when the next class will be makes is hard to plan my time" (Student 74)

To sum up, human contact (learner-learner and learner-instructor) and clarity of information can implement the quality of distance learning; if implemented, these two elements could contribute to offering distance quality learning in possible future EMI courses.

\section{Interaction}

The respondents complained about the quality of interaction and engagement in their EMI classes, especially when education had to suddenly switch to the online modality during the outbreak. They identified several obstacles to the effectiveness of interaction, which included the general low levels of English proficiency and digital expertise, the lectures mode and technical problems.

Overall, the majority of the respondents (91\%) confirmed that English was the main language used by students and lecturers in both face-to-face and online EMI classes. Few students only argued that they occasionally switched to Italian to better express themselves, presumably the Italian ones. However, the low levels of English proficiency, both of students and lecturers, identified by the respondents as a major obstacle to interact in class is one of the biggest challenge of the EMI environment because it may hinder the quality of education and interaction. Although the entry requirements set to enrol in EMI degree programmes in "Medicine and Surgery" and "Nursing" range from B1 to B2, according to the CEFR (Common European Framework of Reference for Languages) (Cicillini, forthcoming; Cicillini (bis), forthcoming), some students complained about poor learner-instructor and learner-learner interaction. In their view, this was mainly due to low and different levels of English proficiency and confirmed by the fact that some were not able to express themselves correctly and fluently.

"The lecturers spoke English poorly, and with strongly marked accents" (Student 2) 
"My English is at a higher level compared to most of the people around me, both students and professors. I think my English might have even deteriorated" (Student 11)

Others claimed that the quality of interaction worsened in the shift to the online emergency education mainly because of issues related to the digital modality. They complained about internet connection problems (72\%) faced, technical problems (30\%) and the lack of a personal device for studying (10\%) which have undoubtedly made communication and interaction more difficult. In addition, they mentioned the loss of clear instructions and of a standardization of lessons among the disciplines taught. As a matter of fact, no common strategy was adopted to offer EMI lectures, as some lessons were offered through the live modality, occasionally recorded and uploaded in the lecturer's personal page, and others were encapsulated into pre-recorded PowerPoint presentations. The data shows that especially in the pre-recorded lessons, interaction was quite challenging because the only ways allowed were the use of forums (20\%) and emails (60\%). In the live sessions instead, the respondents argued that they sometimes used the chats (49\%), with or without the lecturers' permission, but that they did not feel at ease to interrupt the lessons and ask for explanations and feedback. As a consequence, $66 \%$ of the students argued to have interacted with their classmates (learner-learner interaction) and asked them for help instead of interacting with the lecturers. Below, some comments given by the respondents:

"Those that gave us the lessons were only a registration of slides with audio. Only two professors out of 10 had a live session for questions. Nothing was positive about it" (Student 13).

"I only had few live lessons with the professors. Mostly, study materials (ppt, documents, reference books) were posted in Moodle or in Google classroom" (Student 67).

"There was no interaction, just pre-recorded lectures" (Student 55).

"There was no interaction with both the lecturer and the classmates. The only interactions were via chat in both cases. One professor encouraged oral interactions during the lecture but the noise of everyone's mics interfered" (Student 98).

To sum up, according to the respondents, both learner-learner and learner-instructor interaction in face-to-face classes is more meaningful and dynamic than in the online modality probably because they are more used to ask and receive feedback immediately and feel more comfortable with human interaction. Instead, the sudden shift to the digital 
Cicillini, S., \& Giacosa, A.

Communication and Interaction from Face-to-Face to Online EMI Degree Programmes in the Students' Perspective - A Case Study

environment has proved to be very challenging, presumably because of the lack of time to design the courses in the online modality. This may have been also due to the students and lecturers' poor digital skills and the various technical problems faced during the outbreak. Instead, the problem of English proficiency still remains a big issue to address, both in faceto-face and future online classes. These findings suggest that in the students' perspective, interaction in EMI classes is an essential part of the course programme and consequently a detailed planning of the objectives and outcomes seems necessary to guarantee higher educational quality and encourage the implementation of future online EMI programmes throughout the country.

\section{Conclusions}

This study has presented the results of a questionnaire addressed to students enrolled in the academic year 2019/2020 in EMI degree programmes in "Medicine and Surgery" and "Nursing" in Italy. Their perceptions, opinions and expectations have been investigated in order to explore how communication and interaction have changed from the face-to-face to the online modality during the pandemic (RQ1) and to identify possible best practices for future online EMI programmes (RQ2).

As regards RQ1, the sudden shift from the face-to-face to the online modality has been problematic for several reasons but mainly because the university community has had very little time to adapt to the new learning environments and needs. While communication and interaction have always been very challenging in face-to-face EMI contexts, they have exacerbated in the digital modality because of the inadequate proficiency for handling communication effectively through the medium of English, of low digital expertise and various technical problems.

Indeed, whereas in the face-to-face modality communication and interaction used to take place traditionally in class and in person through questions, debates and immediate feedback, new strategies and tools were introduced in the online modality during the outbreak. Although during the emergency the sudden introduction of certain digital platforms and tools soon became the only way to learn and keep in touch with professors and classmates, not all the stakeholders involved were able to use them efficiently. As a matter of fact, some respondents claimed that many lecturers and students did not know how to use certain digital tools and did not receive enough instructions to maximize their full potential. Therefore, digital training and support, together with clearer instructions, would be necessary in case of future EMI online programmes. Although ERE education has suddenly replaced the traditional education without notice, it has also provided new insights into interaction and communication in EMI online courses and how they could be improved in future learning environments. Regarding the RQ2 concerning the lessons 
Cicillini, S., \& Giacosa, A.

Communication and Interaction from Face-to-Face to Online EMI Degree Programmes in the Students' Perspective - A Case Study

learned from the sudden transition from in-person EMI classes to ERE, four main aspects can be identified. First data shows the need for specific training on best practices to deal with communication and interaction. English proficiency is an issue of concern for EMI courses (both in-person and online) and according to the respondents more resources should be put in place to increase lecturers' and students' language skills. Apart from English proficiency and knowledge of EMI pedagogy, lecturers should rely on effective tools to implement interaction among students and be trained on how to use them successfully. For example, students could benefit from interacting in small groups in safe digital rooms, but on the one hand not all the platforms offer this option and, on the other hand, not all the lecturers are familiar with them. Second, this study has shown that the lack of clear and binding guidelines on how to teach lessons has impacted the quality of communication: that the standardization of the classes format should be a top priority in students' opinion. As a consequence, confusion can be minimized by adopting similar modes in the delivery of lessons. Third, students would like to have more opportunities to interact with their lecturers: more office hours could be useful, but they could increase the burden for lecturers. Peer tutoring with older students could help students to better understand the contents and to increase human contact and interaction. Finally, the quality of the interaction should be improved: some students lamented the difficulty of interacting during classes because of the background noise due to activated microphones. Although some lecturers encouraged students to unmute their microphones and speak their minds or voice their concern, they had to mute them because of the annoying echo. Faculties should invest in effective tools to promote interaction during classes, which could allow students to interact more.

To sum up, ERE has proved that EMI courses can be taught online; however, some key issues still need to be tackled to rely on effective interaction and communication in online EMI classes. Further research, training and investments can make the difference.

\section{References}

Bali, M. (2020, May 13). Literacies Teachers Need During Covid-19. Al-FanarMedia [Blog post]. https://www.al-fanarmedia.org/2020/05/literacies-teachers-need-during-covid$19 /$

Borsetto, E., \& Schug, D. P. (2016). English Support to Academic Staff: A Pilot Study at the Department of Management. Annali di Cà Foscari. Serie Occidentale, 50, 9-28.

Bozkurt, A., Xiao, F., Jung, I., \& Vladimirshi, V. (2020). A global outlook to the interruption of education due to COVID-19 Pandemic: Navigating in a time of uncertainty and crisis. Asian Journal of Distance Education, 15(1). 
Broggini, S., \& Costa, F. (2017). A survey of English-medium instruction in Italian higher education. Journal of Immersion and Content-Based Language Education, 5(2), 238264.

Cicillini, S., \& Salusso, D. (2019). Content interaction in online university courses: the start@unito project. Proceedings of the HEAD'19, $5^{\text {th }}$ International Conference on Higher Education Advances. Editorial Universitat Politècnica de València, 1197-1205. https://doi.org/10.4995/HEAD19.2019.9449

Cicillini, S. (forthcoming). English language entry requirements in EMI degree programmes at bachelor level in Italy.

Cicillini, S. (bis) (forthcoming). EMI degree programmes at master level in Italy: the English entry requirements.

Costa, F., \& Coleman, A. J. (2013) A survey of English-medium instruction in Italian higher education. International Journal of Bilingual Education and Bilingualism, 16(1), 3-19, doi: 10.1080/13670050.2012.676621

Dafouz Milne, E., \& Sánchez García, D. (2013). ‘Does everybody understand?’ Teacher questions across disciplines in English-mediated university lectures: An exploratory study. Language Value, 5(1), 129-151

Drljača Margic, B., \& Vodopija-Krstanovic, I. (2018). Language development for English medium instruction: Teachers' perceptions, reflections and learning. Journal of English for Academic Purposes, 35, 31-41.

Macaro, E., Curle, S., Pun, J., An, J., \& Dearden, J. (2017). A systematic review of English medium instruction in higher education. Language Teaching, 51(1), 36-76.

Moore, M. G. (1989). Three types of interaction. The American Journal of Distance Education, 3(2), 1-6.

Pulcini, V., \& Campagna, S. (2015). Internationalisation and the EMI controversy in Italian higher education. In S. Dimova, A. K. Hultgren, C. Jensen (Eds.), EnglishMedium Instruction in European Higher Education (pp.65-87). Berlin: Mouton De Gruyter.

TAEC EMI Handbook (2019). TAEC Erasmus+ project (2017-2020).

Wächter, B., \& Maiworm, F. (2014). English-taught programmes in European Higher Education: The state of play in 2014. ACA papers on international cooperation in education. Bonn: Lemmens. 